Plasma Phys. Control. Fusion 49 (2007) 1391 - 1408

\title{
Application of a bandpower correlation method to the statistical analysis of MHD bursts in quiescent Wendelstein-7 AS stellarator plasmas
}

\author{
G. Pokol ${ }^{1}$, G. Por ${ }^{1}$, S. Zoletnik ${ }^{2}$, W7-AS team ${ }^{3}$ \\ ${ }^{1}$ Department of Nuclear Techniques, Budapest University of Technology and \\ Economics, Association EURATOM, Mủegyetem rkp. 9., H-1111 Budapest, Hungary \\ ${ }^{2}$ KFKI-RMKI, Association EURATOM, Pf. 49, H-1525 Budapest, Hungary \\ ${ }^{3}$ Max-Planck-Institut für Plasmaphysik, Association EURATOM, D-85748 \\ Garching, Germany \\ E-mail: pokol@reak.bme.hu
}

\begin{abstract}
This paper presents the analysis of Mirnov coil data from pure electron-cyclotron resonance heated Wendelstein-7 AS discharges with transient magnetohydrodynamical (MHD) mode activity in otherwise MHD inactive plasmas. Amplitude modulations were found on the $100 \mu s$ time scale, expected to originate from transient changes of the plasma pressure profile by anomalous transport events. The analysis was done using our custom developed bandpower correlation method, which combines short-time Fourier transform for the decomposition of the signal with a correlation analysis to quantitatively characterize the statistical connection between the modulation in the power of different MHD modes. The effect of a confinement transition (near a rational rotational transform) on the correlation between the power modulation of different frequency modes has been studied. As high as $50 \%$ correlation has been found between different frequencies in 'bad confinement' shots with enhanced transient transport, while for 'good confinement' shots no significant correlation was observed.
\end{abstract}




\section{Introduction}

Bursts in Mirnov coil signals were frequently observed on the Wendelstein 7-AS (W7AS) stellarator in otherwise magnetohydrodynamically (MHD) inactive plasmas. In this paper, we analyze data from pure electron-cyclotron resonance heated (ECRH) discharges with a moderate heating power. Under such conditions neither fast ions (like in Ref. [1]) nor steep pressure gradients are present to drive MHD modes. We believe these magnetic bursts to be rapidly damped MHD modes excited by some transient deviation of the plasma from the MHD equilibrium.

Previously, it was found [2] that the changes in the root mean square (RMS) amplitude of the Mirnov coil signals were connected statistically to the modulation in the millimeter-scale density fluctuation amplitude on the $100 \mu \mathrm{s}$ time scale measured by the LOTUS collective laser scattering system [3]. This indicates that transient MHD modes might have some role in the anomalous transient transport processes determining plasma confinement properties. Correlation between Mirnov coil RMS amplitude and other transport related quantities were also found [4]. Further observation were that transient MHD modes could also be seen as quasi-periodic edge density fluctuations detected by Lithium-beam emission spectroscopy (Li-BES) [5], they were always localized radially inside the last closed flux surface (LCFS) [5], they could have a poloidal structure [6], and they were rotating in the electron diamagnetic drift direction [6].

In order to analyze the relevance of these phenomena to transport we investigate discharges near to the $t=1 / 3$ rotational transform, where about a twofold change in confinement time can be seen as a response to a small $t$ change. This effect is believed to be the manifestation of the rotation transform sensitivity of anomalous transport in W7-AS. [7]

In this paper we analyze the time-frequency structure of the transient MHD modes in further detail using the custom-developed bandpower correlation method, which is also to be described. Our bandpower correlation method can be used, whenever the measured signal contains contributions of different origin separated in frequency. In such cases, the signal can be decomposed into its components. By investigating the correlations between these signal component amplitudes, one can get information about the functioning of the physical system. Common source of fluctuations having different characteristic frequencies, energy transfer between eigenmodes, or spatial correlations can be revealed in this way.

It will be shown that the bandpower correlation method is the right choice for the analysis of the Mirnov coil signals in the experiment series processed in this paper. After a short introduction on the shots analyzed, detailed information on the poloidal Mirnov coil ring used in the measurements is given in Section 2.1 and the resulting signals are introduced in Section 2.2. The bandpower correlation method described in Section 3 is a signal processing method combining two fields of signal processing: time-frequency analysis and correlation analysis. In the first step the signal is decomposed by a shorttime Fourier transform (STFT) [8]. Arguments for the selection of the transform and the 
transform properties are presented in detail in Section 3.1. In the second step, frequency bands are selected and bandpowers are calculated. A few potential methods and some basic properties of the resulting bandpowers are discussed in Section 3.2. As the last step of the method, cross-correlation functions are calculated between bandpowers in Section 3.3. The method is compared to sister methods in Section 3.4. The bandpower correlation method has been tested by processing simulated signals, and the limitations of the method were explored, which are presented in Section 4. Results using the real signals and a discussion of the underlying physics are presented in Section 5. Finally, conclusions are drawn in Section 7.

\section{Experimental setup, signal properties}

In this paper the W7-AS shot series \#52123-52175 is processed, which was dedicated to the investigation of the confinement transition around $t_{a}=1 / 3$. This experiment consisted of $440 \mathrm{~kW}$ ECRH heated, $B_{T}=-2.5 \mathrm{~T}$, net current free 'good confinement' (at $t_{a} \approx 0.35$ ) and 'bad confinement' (at $t_{a} \approx 0.36-0.37$ ) discharges at four different plasma densities. At high densities there was a twofold change in energy confinement as a response to this small change in the magnetic geometry. Toward lower densities the difference in the confinement properties decreases, and at the lowest density shots of this series it vanishes. We have six identical shots of each type and a nice flat top which gives us reasonably good statistics. Basic properties of the shots at the flat top can be found in Table 1.

Table 1. Basic data of the shots analyzed

\begin{tabular}{|l|c|c|c|c|c|c|}
\hline Shot number & $\begin{array}{c}\text { Confine- } \\
\text { ment }\end{array}$ & $\iota_{a}$ & $\begin{array}{c}W_{\text {dia }} \\
(\mathrm{kJ})\end{array}$ & $\begin{array}{c}\int n_{e} d l \\
\left(10^{19} \mathrm{~m}^{-2}\right)\end{array}$ & $\begin{array}{c}T_{e}(0) \\
(\mathrm{keV})\end{array}$ & $\begin{array}{c}\tau_{e} \\
(\mathrm{~ms})\end{array}$ \\
\hline$\# 52123-28$ & 'good' & 0.354 & 3.5 & 0.95 & 1.7 & 8.0 \\
\hline$\# 52129-34$ & 'bad' & 0.373 & 2.4 & 0.95 & 2.0 & 5.5 \\
\hline$\# 52135-40$ & 'bad' & 0.373 & 3.7 & 1.8 & 0.9 & 8.4 \\
\hline$\# 52141-46$ & 'good' & 0.350 & 6.8 & 1.9 & 1.1 & 15.5 \\
\hline$\# 52147-52$ & 'good' & 0.346 & 8.4 & 2.7 & 0.9 & 19.1 \\
\hline$\# 52153-58$ & 'bad' & 0.364 & 4.6 & 2.6 & 0.6 & 10.5 \\
\hline$\# 52164-69$ & 'good' & 0.354 & 1.4 & 0.4 & 2.4 & 3.2 \\
\hline$\# 52170-75$ & 'bad' & 0.373 & 1.4 & 0.4 & 2.8 & 3.2 \\
\hline
\end{tabular}

A few shot parameters of a 'good confinement' (\#52147) and a 'bad confinement' (\#52153) shot from the highest density discharges are plotted in Figure 1. These two shots had the same density, heating $(\mathrm{ECRH})$, toroidal magnetic field $\left(B_{0}\right)$, vertical magnetic field $\left(B_{z}\right)$ and plasma radius $\left(a_{P}\right)$. The main difference was a slight change in the edge rotational transform $\left(\Delta t_{a}=0.018\right)$, which caused a change by a factor of two in the energy content measured by the diamagnetic loop $\left(W_{d i a}\right)$, and in the energy confinement time $\left(\tau_{e}\right)$ accordingly. 
Shot \#52147

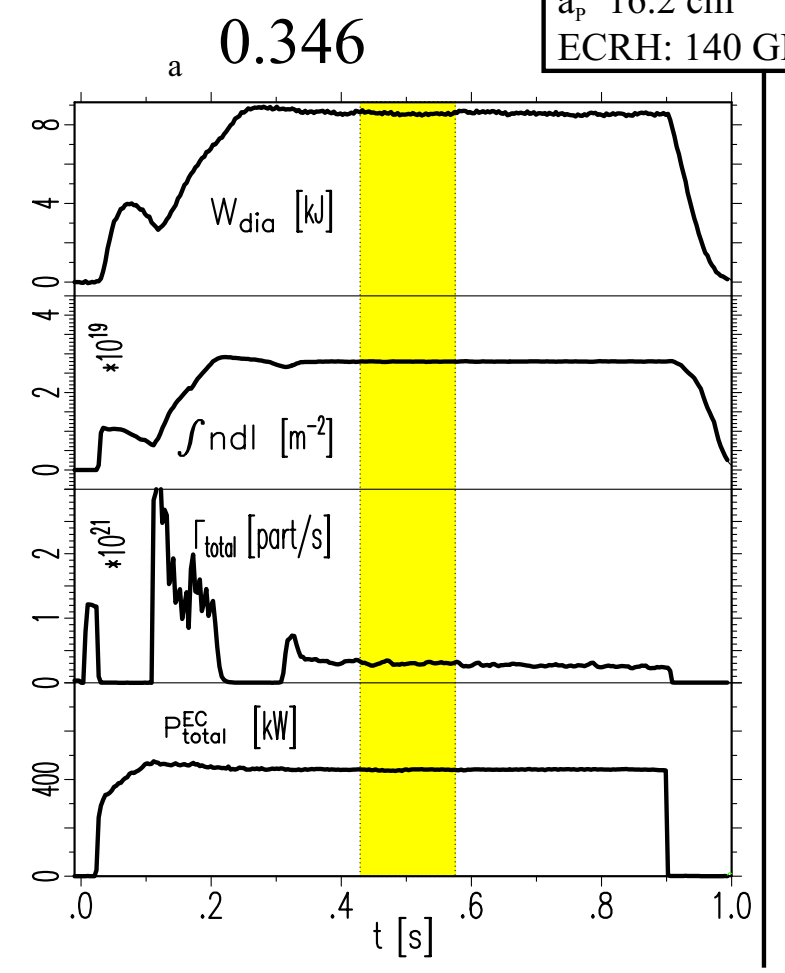

$\mathrm{B}_{0}=-2.5 \mathrm{~T}, \mathrm{~B}_{\mathrm{z}}=-10.2 \mathrm{mT}$
$\mathrm{a}_{\mathrm{P}}=16.2 \mathrm{~cm}$
ECRH: $140 \mathrm{GHz}, \mathrm{X}$ mode
Shot \#52153

$\mathrm{l}_{\mathrm{a}}=0.364$

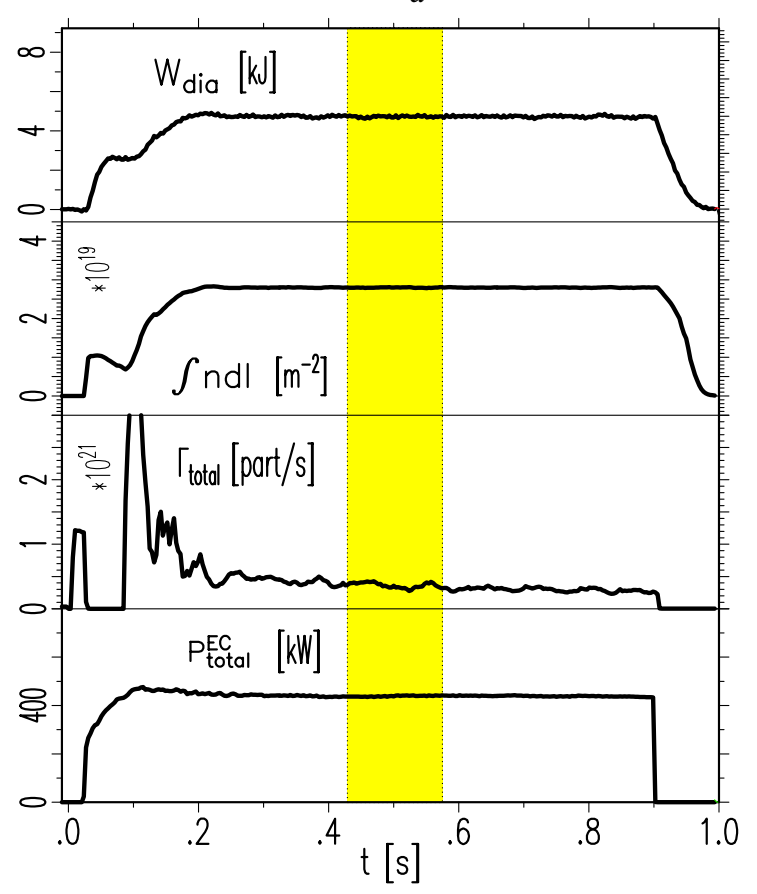

Figure 1. Temporal evolution of some shot parameters (diamagnetic energy, density, gas flux, heating power) for shots \#52147 and \#52153. Time window of the MIR-1 measurement is also plotted as the shaded area.

\subsection{Mirnov coils}

We have analyzed signals of the MIR-1 poloidal Mirnov coil ring at the $\Theta=13.5 \mathrm{deg}$ toroidal position of W7-AS module 5. Poloidal positions of the probes are shown on Figure 2 with magnetic surfaces of the plasma also plotted based on equilibrium MHD fits by the TRANS code. [9] The slightly different transfer functions of the three types of the Mirnov coils used in the array are also plotted, the types of coils are marked with numbers in Italic style. [10] Signals were sampled with $3 \mu s$ sampling time, thus the Nyquist-frequency is $166 \mathrm{kHz}$. However, since the transfer functions exhibit resonance in peaks in the frequency range of $100-150 \mathrm{kHz}$ (see Figure 2), we limited our analysis to components below $100 \mathrm{kHz}$.

\subsection{Signal properties}

MIR-1 coil signals were recorded in two time windows, from which the second one in the middle of the flat top $(0.42-0.58 s)$ has been used for the analysis (see Figure 1 ). Although Mirnov coil signals in these discharges show transient bursts of MHD activity, above the ten millisecond time-scale they have a stationery character, which is exploited during the statistical analysis. 

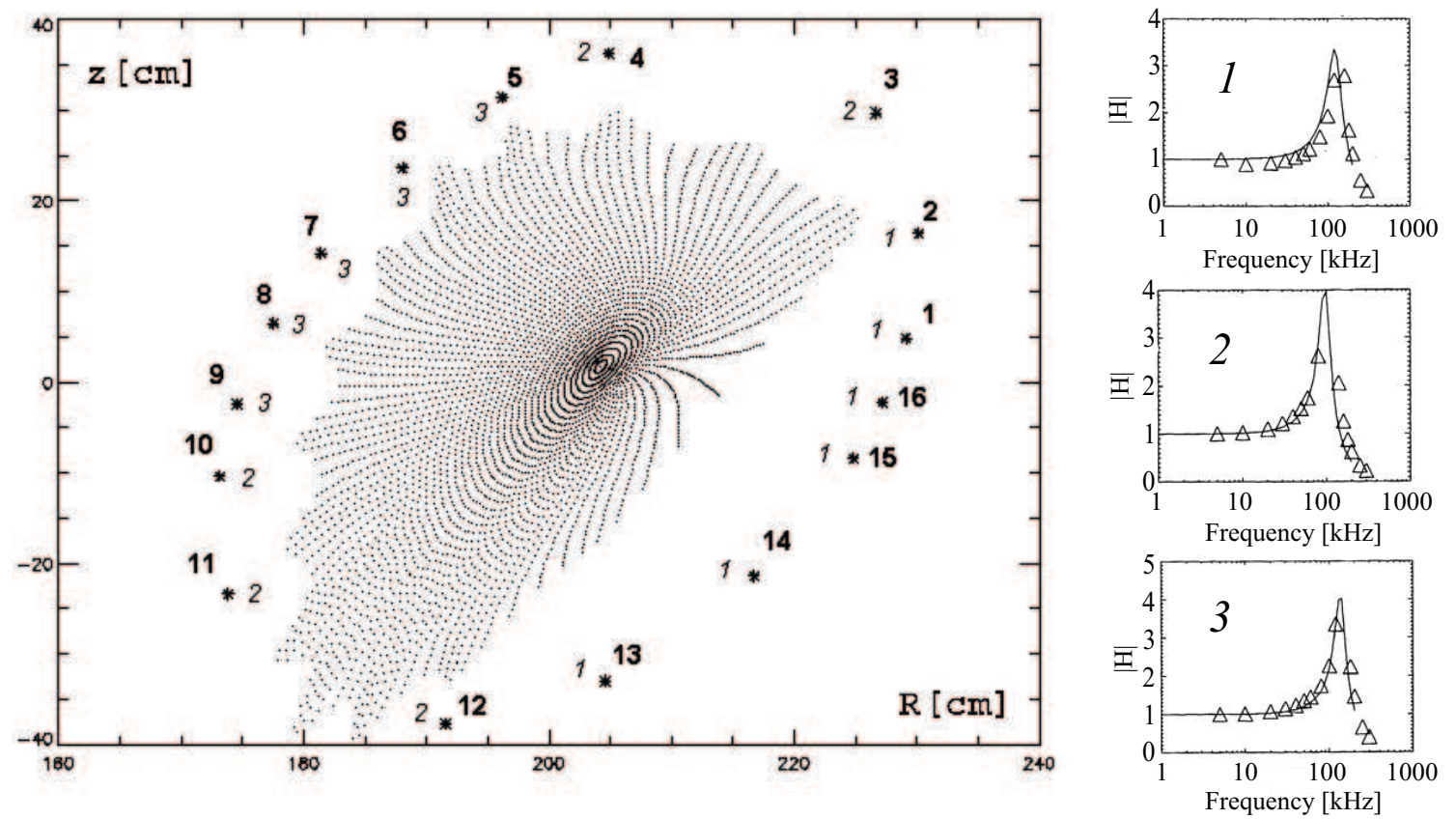

Figure 2. Positions of the coils of the W-7 AS MIR-1 Mirnov coil ring with respect to the plasma and transfer functions of the three types of coils. Bold numbers denote the numbering of the coils, while numbers in Italic denote the type.

Auto power spectral densities (APSDs) of a typical shot (\#52153) are plotted on Figure 3. It can be seen on the APSDs, and it has also been stated previously [11], that most of the power of the Mirnov coil signals is concentrated in a few frequency bands. It is also worth noting that the width of the frequency bands is broad, typically $10-20 \mathrm{kHz}$. This indicates that either the frequency or the amplitude of the modes is changing in time. Indeed, it will be shown that there is no continuous mode activity in these shots. It can also be seen that the height of the different peaks varies largely between the different coils, and in some cases peaks can even be lost in the background broadband noise. This variation can be either due to the fact that coil positions with respect to the plasma are considerably different (as shown on Figure 2) and the plasma shape itself was asymmetric having detailed structure at the edge, or it may be the characteristic of the perturbation itself, like edge originated magnetic perturbations (EOMPs) seen on TFTR [12]. Observation of a well defined spatial structure [6, 13] points to the first explanation suggesting that ordinary MHD modes can appear in such a distorted way in the geometry of our measurement.

\section{The bandpower correlation technique}

Bursts noticed in the time signals, according to the APSDs, have their energy concentrated in characteristic frequency bands. In such cases, the bandpower correlation method can be used to search for statistical connections between the processes producing the signal components at different frequency bands (e.g. MHD modes). 

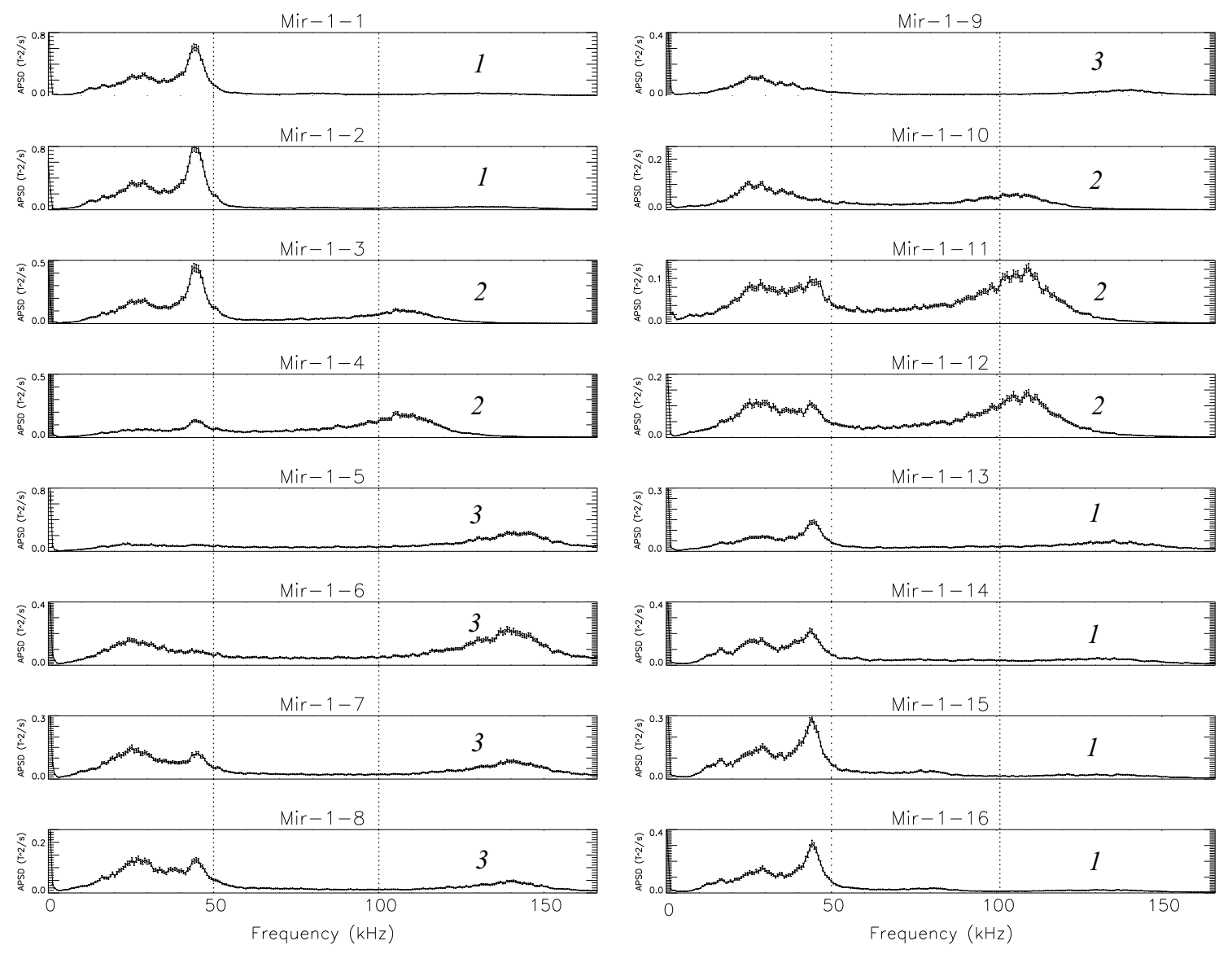

Figure 3. APSDs of the 16 coils of the MIR-1 ring for shot \#52123. (The peaks above $100 \mathrm{kHz}$ are due to resonances in the transfer functions.)

In the first step of the method, the signal is decomposed into time-frequency atoms revealing the time-frequency structure of the individual transient events. Frequency chirps and the envelope of the power modulation can be recovered this way. In our case no frequency chirp could be identified, therefore we became interested in the power modulation of the signal components. The next step is the calculation of the bandpower signals, which represent the power modulation envelope of the different signal components in different frequency bands. These bandpowers are stochastic time-series, they are the starting point for statistical analysis, which is in our case a correlation analysis. Cross-correlation functions (CCFs) between different bandpowers are the end product of the data processing technique, the properties of which reflect features of the physical system subject to measurement.

\subsection{Basic properties of the continuous time-frequency transforms}

The forthcoming summary of the properties of the transform used is based on the work of Mallat [8]. One of the critical points of the bandpower correlation technique is the selection of the transform which produces the time-frequency representation of the signal. The basis of time-frequency analysis of transient signals is most often a 
transform originating from the Wigner-Ville distribution:

$$
P_{V} f(u, \xi)=\int_{-\infty}^{+\infty} f\left(u+\frac{\tau}{2}\right) f^{*}\left(u-\frac{\tau}{2}\right) e^{-i \tau \xi} d \tau
$$

where $f(t)$ is the initial time-series, $u$ is the time and $\xi$ is the frequency coordinate and ${ }^{*}$ denotes the complex conjugate. For single component signals (time-frequency translated Gaussians) this representation gives the exact time-frequency localization of the signal without any parameterization, but in case of more complicated composite signals interference patterns appear allowing negative transform values, which make the interpretation as a time-frequency energy-density distribution very troublesome.

The properties of the Wigner-Ville distribution can be modified by introducing a smoothing:

$$
P_{\Theta} f(u, \xi)=\int_{-\infty}^{+\infty} \int_{-\infty}^{+\infty} P_{V} f\left(u^{\prime}, \xi^{\prime}\right) \Theta\left(u, u^{\prime}, \xi, \xi^{\prime}\right) d u^{\prime} d \xi^{\prime}
$$

where $\Theta\left(u, u^{\prime}, \xi, \xi^{\prime}\right)$ is the time-frequency smoothing kernel. The aim of this smoothing is the suppression of the interference patterns. Different kernels can be selected, but if we aim to produce a meaningful time-frequency energy-density distribution, we have to use linear time-frequency transforms, as the cancellation of interference terms is complete only in this case.

Energy-density distributions of linear time-frequency transforms can be calculated by using the appropriate smoothing kernel on the Wigner-Ville distribution of the signal, but there exists a more efficient and more direct way. These transforms can be calculated by correlating the signal to families of so called time-frequency atoms:

$$
T f(u, \xi)=\left\langle f, g_{u, \xi}\right\rangle=\int_{-\infty}^{+\infty} f(t) g_{u, \xi}(t) d t
$$

where $g_{u, \xi}(t)$ is a time-frequency atom which is a function of time, whose energy is well localized in both time and frequency and $\left\|g_{u, \xi}(t)\right\|=1$. Here, $u$ and $\xi$ are the time and frequency indexes of the atom identifying its position on the time-frequency plane. The energy-density distributions can then be calculated by taking the absolute value squared:

$$
P_{T} f(u, \xi)=|T f(u, \xi)|^{2}
$$

The most important properties of a time-frequency atom are its center in time $u=\int_{-\infty}^{+\infty} t\left|g_{u, \xi}(t)\right|^{2} d t$, its center in frequency $\xi=\frac{1}{2 \pi} \int_{-\infty}^{+\infty} \omega\left|\hat{g}_{u, \xi}(\omega)\right|^{2} d \omega$, its extent in time $\sigma_{t}=\left(\int_{-\infty}^{+\infty}(t-u)^{2}\left|g_{u, \xi}(t)\right|^{2} d t\right)^{\frac{1}{2}}$ and its extent in frequency $\sigma_{\omega}=$ $\left(\int_{-\infty}^{+\infty}(\omega-\xi)^{2}\left|\hat{g}_{u, \xi}(\omega)\right|^{2} d \omega\right)^{\frac{1}{2}}$, where $\hat{g}$ denotes the Fourier-transform of $g$. The timefrequency extent of the atom is bounded from bellow by the uncertainty principle [8]:

$$
\sigma_{t} \sigma_{\omega} \geq \frac{1}{2}
$$

where equality holds for the Gabor-atom:

$$
g_{u, \xi}(t)=\frac{1}{\sqrt{\sqrt{2 \pi} \sigma_{t}}} e^{i \xi t} \exp \left\{-\frac{(t-u)^{2}}{2 \sigma_{t}^{2}}\right\} .
$$


In order to produce an invertible transform, the time-frequency atom family used in the transform has to cover the whole time-frequency plane. This can be achieved by using an orthogonal set of atoms, which gives the traditionally used discrete transforms, or by using a set of atoms with significant overlaps, which approximates the continuous transforms.

Until recent times, discrete representations were preferred over continuous ones in signal analysis. This was partly due to the fact that orthogonal transforms produced a statistically independent set of transform values, while continuous transforms produced transform values connected through a reproducing kernel, and partly due to the lack of sufficient computation power to calculate continuous transforms. However, in the analysis of transient signals all the disadvantages of the continuous transforms are overcome by their advantage of being time-shift invariant. While time-shift invariance is not important in the analysis of stationary stochastic signals, it is critical in the analysis of transient signals.

According to the generation method of the family of time-frequency atoms used in the linear continuous transforms, two basic types can be specified: the frequencyshift invariant short-time Fourier transform (STFT) and the affine invariant continuous analytical wavelet transform (CWT). In the last step of the analysis frequency-shift invariance will be important, so we have decided to use STFT. Reasons for this selection are presented in more detail in Section 3.3.

The family of STFT atoms is generated by shifting an atom in time and frequency (and modifying the phase by $e^{i \xi u}$ ):

$$
g_{u, \xi}(t)=e^{i \xi t} g(t-u),
$$

where $u$ is the time-shift, $\xi$ is the shift in frequency and $g(t)$ is the normalized window function. The corresponding energy-density distribution defined by (4) is called spectrogram. The smoothing kernel in (2) is simply given by the time- and frequencyshifted versions of the Wigner-Ville distribution of the window function:

$$
\Theta\left(u, u^{\prime}, \xi, \xi^{\prime}\right)=\Theta\left(u^{\prime}-u, \xi^{\prime}-\xi\right)=P_{V} g_{u, \xi}\left(u^{\prime}, \xi^{\prime}\right),
$$

which is a convolution smoothing kernel making the spectrogram a member of the shiftinvariant Cohen's class of time-frequency distributions [14].

For STFT several shapes can be selected for the time-frequency atoms. We have used Gabor-atoms (6), which choice means Gaussian-window for $g(t)$. This is the original form of the STFT also called as Gabor-transform [15]. The main advantage of the Gabor-atom is its energy-distribution being smooth in both time and frequency. This property will be utilized in the forthcoming steps of the analysis. The Gaboratom also has the minimum possible time-frequency extent defined by (5). The most important disadvantage of the Gabor-atom is its lack of compact support. Discrete transforms are typically calculated by performing fast Fourier transforms (FFTs) on blocks of data weighted by window functions. This means that the Gabor-atom has to be truncated and it looses its most appealing properties. Furthermore, it is not possible to generate a discrete orthogonal basis from Gabor-atoms in which the signal representation 
is stable [8]. In the continuous case, however, the price has to be paid in the amount of computation necessary to calculate the transform, but all special properties can be preserved. For Gabor-atoms the (8) smoothing kernel takes the following simple form:

$$
\begin{aligned}
\Theta\left(u^{\prime}-u, \xi^{\prime}-\xi\right) & =\left|g_{u, \xi}\left(u^{\prime}\right)\right|^{2}\left|\hat{g}_{u, \xi}\left(\xi^{\prime}\right)\right|^{2} \\
& =\frac{1}{\pi} \exp \left(-\frac{\left(u^{\prime}-u\right)^{2}}{\sigma_{t}^{2}}\right) \exp \left(-\sigma_{t}^{2}\left(\xi^{\prime}-\xi\right)^{2}\right)
\end{aligned}
$$

Having to deal with discrete time signals, the continuous transform has to be discretized. This is achieved by replacing the integrals by sums and discretizing all variables with the smallest possible steps. That is the $\Delta t$ sampling time for the time variable and the $\Delta f=\frac{1}{2 N \Delta t}$ for the frequency variable, where $N$ is the length of the data vector. In order to save computation time, discretization can be done on a more sparse grid, but significant overlaps giving a redundancy factor (lower frame boundary) of at least 4 has to be sustained. Ref. [8] gives an overview of frame theory.

The only thing which still has to be decided upon is the right balance between the time and the frequency resolution. This has to be done by choosing a transform parameter that is the length of the time window for STFT. It can be stated in general, that the time-frequency resolution of the transform has to fit that of the signal itself. We know from previous studies that transient MHD modes seen on W7-AS have a characteristic duration of about $100 \mu s$ [5], so the window of the STFT has to be selected accordingly. There are, however, other considerations, which are in close connection with the next step of the analysis that is the calculation of bandpowers, and therefore considerations regarding this issue will also be discussed in the next section.

\subsection{Definition of the bandpowers}

Although it is very convenient to qualitatively characterize the time-frequency structures in a signal by viewing its spectrogram, a quantitative analysis demands further processing. The next task is to extract the information of interest from this extremely redundant signal representation. In our case, the information of interest is the power modulation of the i-th characteristic frequency, which are here approximated by the so called bandpowers.

The bandpower signal is calculated from the continuous time-frequency energydensity distribution by the following definition:

$$
B_{i}(t)=\int_{\omega_{i, l}}^{\omega_{i, u}} P_{T} f(t, \xi) d \xi
$$

where $\omega_{i, u}$ and $\omega_{i, l}$ are the upper and the lower frequency limits of the $B_{i}(t)$ bandpower. Band limits have to be determined using a priori knowledge of the band limits of the original band-limited components of the system or by some measured signal properties. The simplest technique is the selection of different peaks of the APSD of the signal.

Selection of the band limits has to be in accordance with the time-frequency resolution of the transform. The most important consideration is that the crosstalk 
between the different frequency bands due to the (2) smoothing has to be minimized. The applied Gaussian-window has the advantage that the frequency projection of the (9) smoothing kernel decreases monotonically away from the center. The crosstalk between bandpowers can be calculated exactly, but often it is convenient to select band limits so that the distance between the closest bands is much larger than the frequency extent of the atoms:

$$
\min _{i}\left[\omega_{i+1, l}-\omega_{i, u}\right] \gg \sigma_{\omega}\left(=\frac{1}{2 \sigma_{t}}\right)
$$
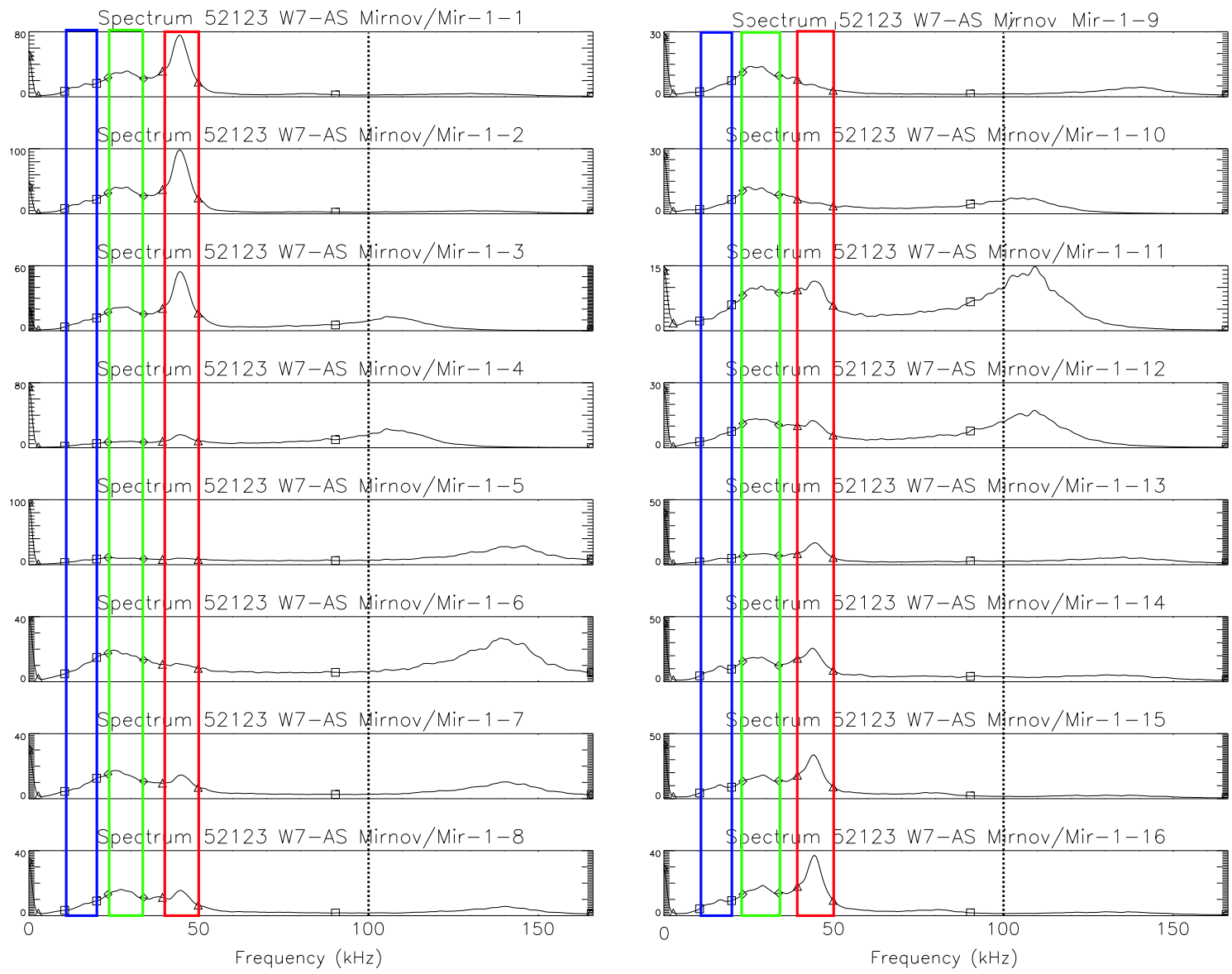

Figure 4. Spectra calculated from the STFT of the 16 Mirnov coil signals of shot \#52123. This plot helps the selection of frequency bands in accordance with the timefrequency resolution of the transform.

A very useful tool that helps the correct selection of the band limits, is the spectrum of the signal calculated by integrating the spectrogram in time. As it can be seen on Figure 4, this picture shows the effect of the (2) smoothing (with $\sigma_{\omega}$ scale) on the spectrum automatically, and allows us to easily select the band limits in accordance with condition (11).

The price which has to be paid for the good frequency resolution is a degradation of the time resolution determined by the (5) uncertainty principle. If the selected frequency 
band is wider than the original bandwidth of the physical process, the bandpower signal approximates the power variation of the band limited process:

$$
B_{i}(t) \approx C \int_{-\infty}^{+\infty} b_{i}\left(t^{\prime}\right) \exp \left(-\frac{\left(t^{\prime}-t\right)^{2}}{\sigma_{t}^{2}}\right) d t^{\prime},
$$

where $b_{i}(t)=\left|f_{i}(t)\right|^{2}$ is the instantaneous energy of the $f_{i}(t)$ signal component and $C=\frac{1}{\sqrt{\pi} \sigma_{t}}$ is the normalizing constant of the kernel. The marginal integral over frequency of the (1) Wigner-Vile distribution of the $f_{i}(t)$ signal component would have produced the exact $b_{i}(t)$ [8]. The smoothing in (12) originates in the (2) time-frequency smoothing with the (9) smoothing kernel. This smoothing has been introduced to eliminate the interference terms and therefore make the separation into bandpowers possible.

Having finite signal lengths, border effects cannot be circumvented. There are several methods to treat the borders, but some effects remain anyway. Therefore it is the most appropriate choice to just truncate the affected part of the bandpowers. The severity of the border effects can be calculated from (12) as a function of distance from border. The place of truncation can be decided upon by negotiating the clarity of the method to the available length of the signal. Truncating the bandpower at $3 \sigma_{t}$ distance from the end of the signal ensures that over $99.9 \%$ of the weight of the smoothing kernel located at the border covers the measured time region.

An alternative to using bandpowers for the extraction of the power modulation of some eigenfrequencies could be based on the method suggested in [16]. The instantaneous amplitude of a signal component can be obtained following the temporal variation of the height of the ridges of the time-frequency energy-density distribution. This technique is superior to the bandpower technique if signal components are frequency-modulated to such an extent that their frequency bands overlap. However, if amplitude modulation dominates in the signal components over frequency modulation, as in our case, ridges are often lost in the noise at the low amplitude time intervals, and it is clearly necessary to use the bandpowers.

\subsection{Properties of the correlation functions}

If we believe that different bandpowers are due to different physical processes (different eigenmodes, etc...) which may be statistically connected through the internal structure of the system, it is worth to make use of correlation analysis between them. Statistical dependencies revealed this way show the functioning of the system.

We use the classical definition of cross-correlation function $\left(\mathrm{CCF}, C_{i j}(\tau)\right)$ between bandpowers $B_{i}(t)$ and $B_{j}(t)$ :

$$
\begin{aligned}
C_{i j}(\tau) & =\frac{R_{i j}(\tau)}{\sqrt{R_{i i}(0) R_{j j}(0)}} \\
R_{i j}(\tau) & =\lim _{T \rightarrow \infty} \frac{1}{2 T} \int_{-T}^{T} B_{i}(t) B_{j}(t+\tau) d t,
\end{aligned}
$$

where $R_{i j}(\tau)$ is the cross-covariance function and $R_{i i}(\tau)$ and $R_{j j}(\tau)$ are the autocovariance functions. 
Since taking the cross-covariance function of two bandpowers is equivalent to taking their product in Fourier-space, it is important that the (2) smoothing does not depend on the frequency. This is ensured by using the frequency-shift invariant STFT for the calculation of the time-frequency energy-density distribution with the (9) smoothing kernel. The analysis was also attempted using CWT, which does not have the frequencyshift invariance property, but interpretation of CCFs was cumbersome, so the final analysis was done using STFT.

It can be shown from (12) and (13) that:

$$
\begin{aligned}
& R_{i j}(\tau)=r_{i j}(\tau) *\left(C^{2} \exp \left(-\frac{2 \tau^{2}}{\sigma_{t}^{2}}\right)\right), \\
& r_{i j}(\tau)=\lim _{T \rightarrow \infty} \frac{1}{2 T} \int_{-T}^{T} b_{i}(t) b_{j}(t+\tau) d t,
\end{aligned}
$$

where $r_{i j}(\tau)$ is the cross-covariance function of the energy of original band limited signal components, and $*$ denotes convolution.

CCFs calculated are presented in Section 5.

\subsection{Comparison with sister methods}

In this section the bandpower correlation method is compared to sister methods mentioned in [17]. Perhaps the closest relative to our method is the amplitude correlation method [18]. The main difference is that, in that method, selecting frequency bands with different bandwidth means that their smoothing introduced by the bandpass filtering will differ, which makes the interpretation of their CCFs difficult.

Other popular methods capable of revealing similar information about a signal are different polyspectrum calculations [17]. Polyspectra can reveal the presence of different order nonlinear interactions between different frequencies through a phase coupling. Phase coupling is not necessary for the bandpower correlation method, as we only compare the power modulation. If the simultaneous power modulation of different modes was caused by common excitation (not nonlinear coupling inside the system), polyspectra would not reveal the statistical connection. Therefore, we can conclude that for these signals, the bandpower correlation unfolds signal properties not revealed by sister methods.

\section{Testing the method on simulated signals}

In order to test the method, we have simulated signals resembling the real measurements. We have constructed a burst-like envelope function with relatively quick rise-time and slower attenuation. Different frequency oscillations were amplitude modulated with a large number of randomly placed and parametrized envelopes. The simulated signal was produced by adding these different frequency signal components and optionally an additive white noise. Signals could be produced with uncorrelated and correlated amplitude modulation and different levels of added noise. 

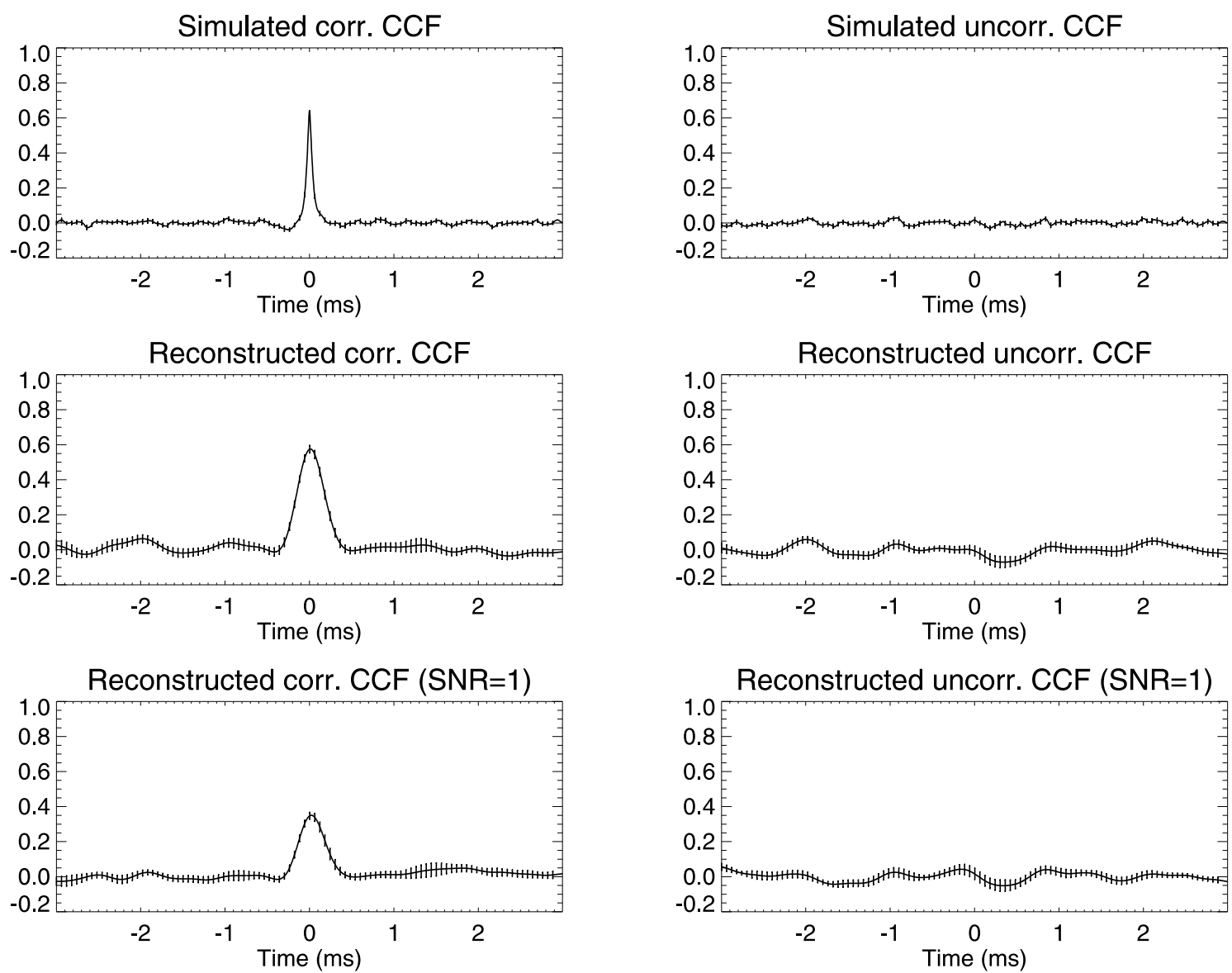

Figure 5. Testing the bandpower correlation method on simulated signals. 1. row: CCFs of the simulated power modulations, 2. row: reconstructed CCFs without added white noise, 3. row: reconstructed $\mathrm{CCFs}$ with $\mathrm{SNR}=1$

We have tried to select the parameters of the simulated signal close the parameters of the real signals. The sampling time was $3 \mu \mathrm{s}$. The length of the bursts varied about $100 \mu s$, they were quite frequent with a largely varying amplitude. Our intention was to test the method for the 'worst case scenario', so we have simulated six modulated frequencies with only $10 \mathrm{kHz}$ between the closest ones. The character of the timefrequency structure of the simulated signals highly resembled that of the real signals seen on Figures 7 and 8 for the uncorrelated and the correlated modulation respectively.

The first row of Figure 5 shows the CCFs of the power modulation of the two closest frequencies ( 25 and $35 \mathrm{kHz}$ ) for the correlated and the uncorrelated case. The next row shows the reconstruction of the CCFs with the bandpower correlation method for the case when no white noise had been added to the signal. The analysis was performed with the same parameters (e.g. $300 \mu$ s long time window) as for the real signals. Error estimation was performed in the following way, making use of the observation that the simulated signal (and the measured signal, as well) shows stationary character above the $10 \mathrm{~ms}$ timescale: The signal was divided into $15 \mathrm{~ms}$ long segments, CCFs were calculated for each segment. The empirical standard deviation calculated from the 
CCFs for each time delay was divided by the square root of the number of segments. This estimation of the standard deviation of the CCF calculated from the whole time interval has been plotted on Figure 5 as error bars. This method was used for the measured signals shown in Section 5, as well.

The correlated case on Figure 5 shows that the smoothing according to (12) makes the correlation-time determination of the bursts practically impossible, but the magnitude of the central peak characterizing the strength of the statistical connection between the power modulation of the different frequency oscillations has been recovered exactly. The uncorrelated case proves that there was no significant crosstalk between the different bandpowers that would have produced a central peak.

The last row of Figure 5 shows CCFs produced by the bandpower correlation method for the simulated signal with added white noise. The amplitude of the noise was chosen to give a unit signal to noise ratio (SNR). $S N R=1$ in this case is to be understood within the band limits that is equivalent to $S N R_{t o t}=0.2$ for the whole signal. While the uncorrelated CCF was unaffected, the correlated CCF has significantly decreased, but the peak around $\tau=0$ is still well seen.

\section{Experimental results}

Mirnov coil signals from the series of plasma discharges presented in Section 2 were analysed using the bandpower correlation technique presented in Section 3. Frequency bands were selected from the time-integrated spectrograms as shown in Section 3.2. Table 2 summarizes the frequency bands for different discharge types.

Table 2. Summary of the results

\begin{tabular}{|l|c|l|c|}
\hline Shot number & Confinement & Frequency bands $(\mathrm{kHz})$ & $C C F(0)$ range (\%) \\
\hline$\# 52123-28$ & 'good' & $10-20,23-34,39-50$ & $0-5$ \\
\hline$\# 52129-34$ & 'bad' & $10-18,20-32,35-50$ & $40-45$ \\
\hline$\# 52135-40$ & 'bad' & $10-32,35-50,60-80$ & $40-55$ \\
\hline$\# 52141-46$ & 'good' & $20-25,28-37,40-50,55-70$ & $0-6$ \\
\hline$\# 52147-52$ & 'good' & $8-13,21-27,31-36,40-45,61-70$ & $-20-20$ \\
\hline$\# 52153-58$ & 'bad' & $10-18,22-45,55-80,90-115$ & $40-55$ \\
\hline$\# 52164-69$ & 'good' & $8-13,18-30,34-45,50-65$ & $7-10$ \\
\hline$\# 52170-75$ & 'bad' & $10-20,25-30,34-42,48-65$ & $50-55$ \\
\hline
\end{tabular}

CCFs have been calculated between all pairs of bandpowers in each shot listed for each Mirnov coil signal. Error estimation was done in the way described in Section 4. We took the mean of the CCFs calculated for the 16 different Mirnov coil signals. Signals measured by the different coils were far from being independent, so we took the mean of their errors also. As these results from the identical shots turned out to be very similar, we took the mean of their CCFs, thus improving signal statistics. In most cases, CCFs between different bandpower pairs showed similar characteristics. Figure 


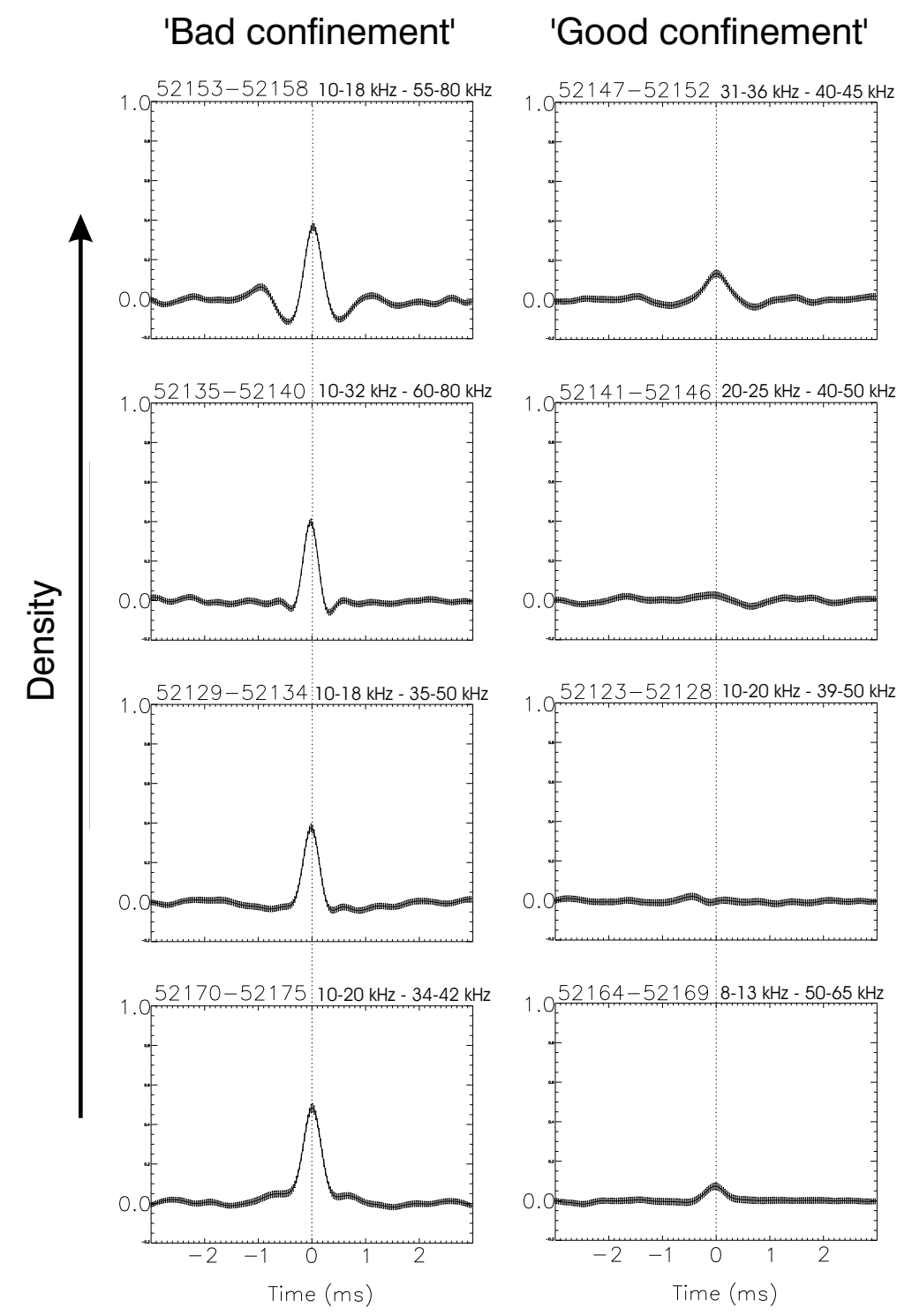

Figure 6. Results of the 8 shot series represented by a typical average CCF each.

6 shows typical mean CCFs, while the last column of Table 2 gives the range of CCF central values for each shot type.

It can be stated that the correlation is significant - about $50 \%$ - for the 'bad confinement' shots and it is insignificant, or at least an order of magnitude less, for 'good confinement' shots. We can conclude that in case of 'good confinement', peaks of bandpower fluctuations appear randomly and uncorrelated at different MHD mode frequencies. In case of 'bad confinement' however, bursts appear randomly, but at the same time at all frequencies, thus they are correlated.

It is worth looking at short sections of the spectrogram to visualize the difference between measurements with high and low bandpower correlation. Figures 7 and 8 show spectrograms of short time intervals of typical 'good' (\#52123) and 'bad' (\#52153) confinement shots respectively. A window length of $300 \mu s$ has been used in both cases. 


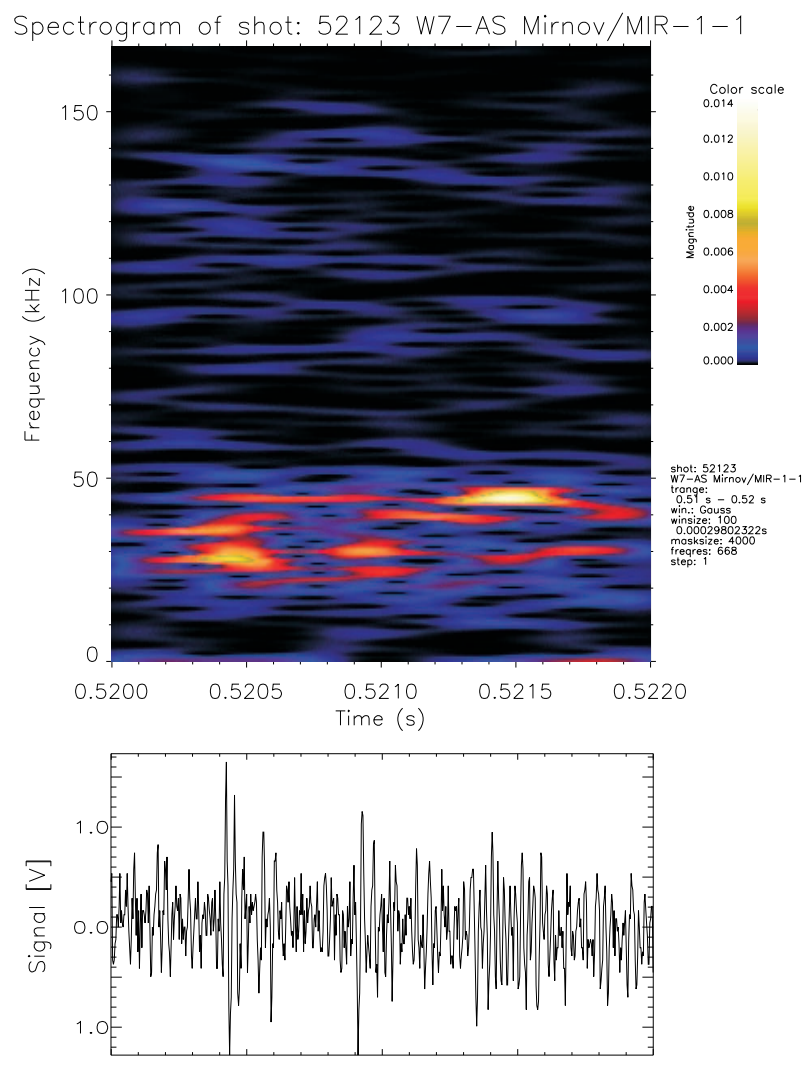

Figure 7. Spectrogram and raw signal of a short segment from the Mirnov coil signal of a typical 'good confinement' shot (\#52123).

It can be seen immediately that their time-frequency structures are very different. On Figure 7 transient MHD modes appearing at $30 \mathrm{kHz}$ and at $45 \mathrm{kHz}$ do not coincide in time. These MHD bursts having well defined frequency are typical of the 'good confinement' shots. The central frequency of the modes changes slightly from burst to burst, but they are organized around characteristic frequencies. As the bursts are very frequent, they often overlap in time, but their temporal power modulation is different. Sometimes two frequencies show up around the same time instance (like at $0.5204 \mathrm{~s}$ ), but the CCFs of the bandpowers, examples of which are shown on Figure 6, showed these to be accidental coincidences.

The 'bad confinement' shot, a short segment of which is presented on Figure 8, shows a completely different picture. Here the bursts are not localized in frequency, components appearing at the same time stretch through the whole frequency range from $10 \mathrm{kHz}$ to $150 \mathrm{kHz}$. Although most of their energy concentrates around characteristic frequencies, modulation of these frequencies occurs simultaneously and it is accompanied by a broadband noise. Figure 8 also shows that although the energy of the burst is not localized in a single frequency band, its spectrum is far from being white. This makes it evident, that these events are not simple Dirac-delta-like spikes or Heaviside-functiontype relaxations. This time-frequency structure is similar to the magnetic fingerprint of the edge localized mode (type III ELM) [19, 20]. In our shots the plasma was in 


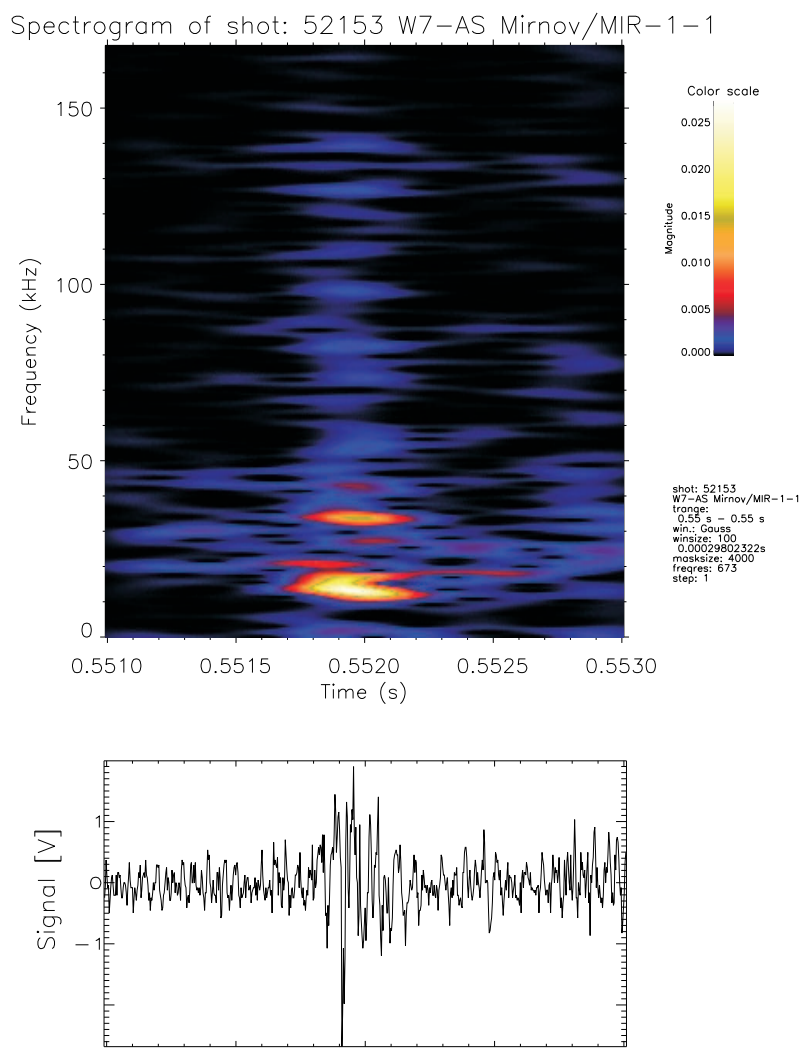

Figure 8. Spectrogram and raw signal of a short segment from the Mirnov coil signal of a typical 'bad confinement' shot (\#52153).

L-mode, so we cannot say that these were real ELMs, but transient transport events showing some characteristics of the ELMs have been previously observed on W7-AS, and they were called ELM-like modes [21].

Looking at Table 2 and Figure 6, shot series \#52147-52152 seems to be an exception from the rule that good confinement shots have low bandpower CCFs. One can clearly see a peak in the average CCF plotted as opposed to the other 'good confinement' shots, which show no correlation. A significant (about 20\%) correlation can be seen between most of the bandpowers. Figure 9 shows that - unlike in 'bad confinement' discharges a -20\% negative correlation can be found between the lowermost frequency band (around $10 \mathrm{kHz}$ ) and all other bands. It indicates that the appearance of activity at the lowest frequencies inhibits activity at all higher frequencies. This causes a positive correlation among all higher bands. Shot series \#52164-52169 also shows an even smaller $(<10 \%)$ - but still significant - correlation. This is not surprising having seen that at this low density difference between the energy confinement in 'good confinement' and 'bad confinement' states also disappears (see Table 1). This is in sharp contrast to shot series \#52147-52152, where the difference is the most pronounced. No signs of negative correlation have been seen in shot series \#52164-52169. 

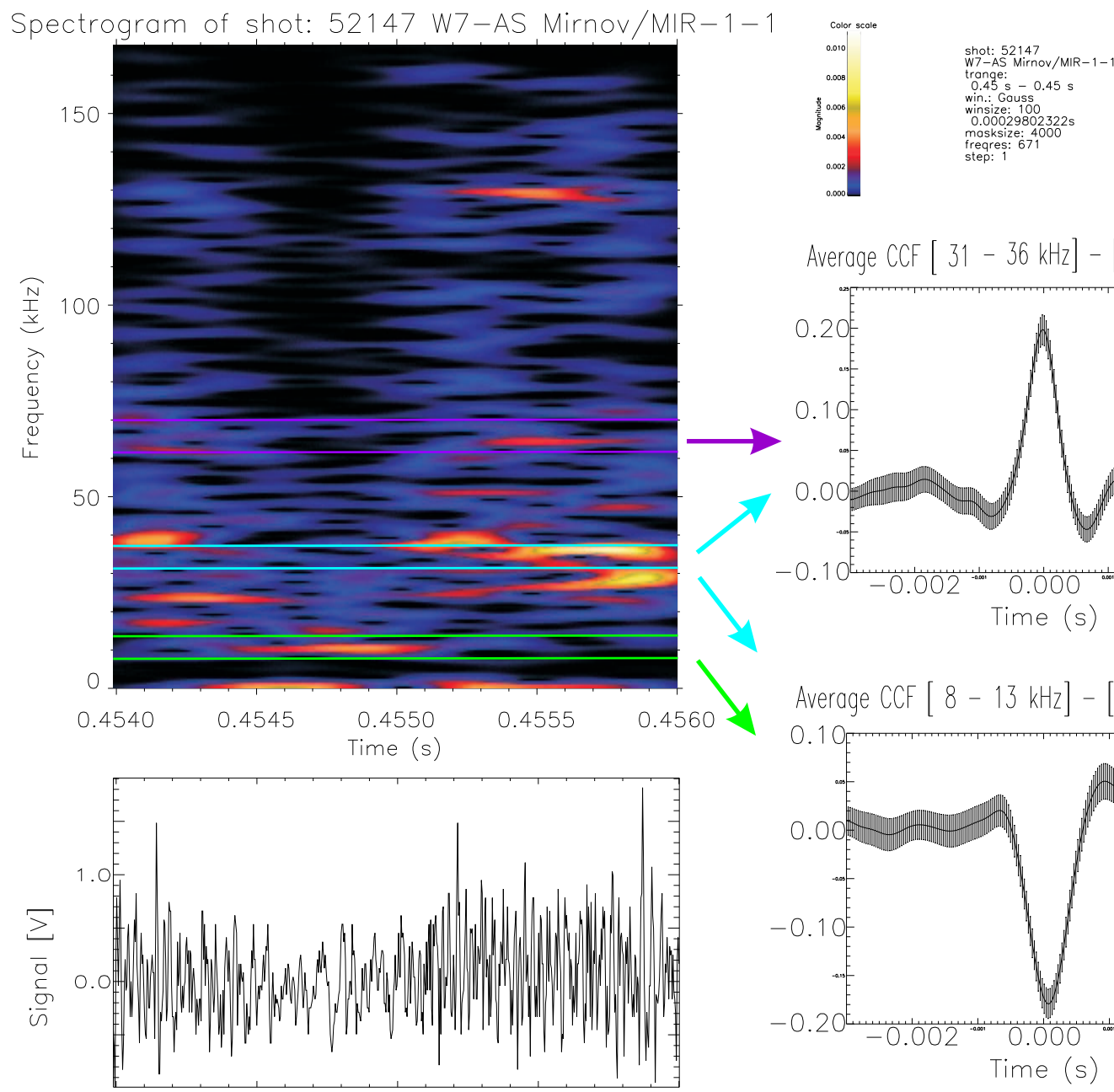

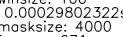

freares:
step: 1

Average CCF $[31-36 \mathrm{kHz}]-[61-70 \mathrm{kHz}]$

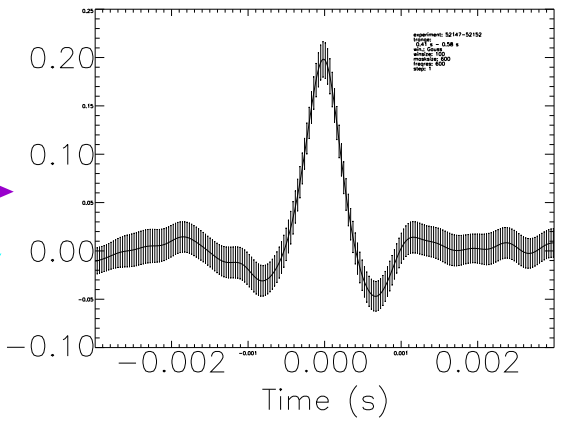

Average CCF $[8-13 \mathrm{kHz}]-[31-36 \mathrm{kHz}]$

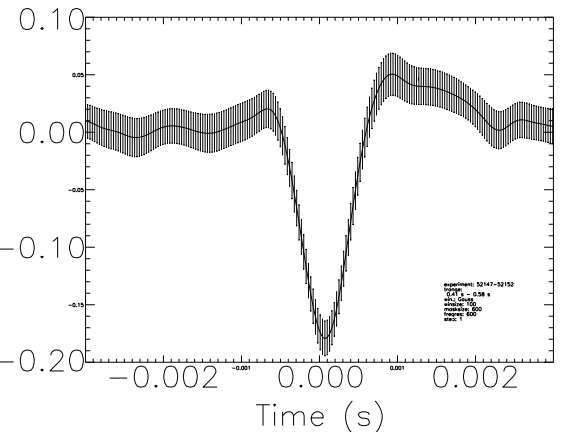

Figure 9. Spectrogram and raw signal of a short segment from the Mirnov coil signal of shot \#52147. Correlation between higher frequency bandpowers has been shown to originate from an anti-correlation with the bandpower around $10 \mathrm{kHz}$.

\section{Discussion}

It is not the intention of this paper to give a full theoretical explanation, but these observations fit well into the model emerged from a broader investigation of transient transport phenomena in W7-AS [4]. There it was shown that the transient MHD mode, flattening in the radial temperature profile (showing the characteristic of an ELM-like mode [21]) and an increase of the mm-scale fluctuation amplitude [2] all belonged to the same phenomenon.

According to our hypothesis, different characteristic frequencies of the modes can be linked to different radial localization through the radial variation of the poloidal flow velocity. This statement is verified by the observation that during a slow transition from 'good confinement' to 'bad confinement' the characteristic frequencies changed gradually, and slower flow velocities resulted in lower frequencies [11]. This way, different frequencies indicate phenomena at different radial locations. 
There are two possible explanations to the strong correlation between transient transport and transient MHD modes: either an MHD instability is causing the rapid radial transport, or the other way around.

Regarding the first possibility, the instability is probably destabilized at some critical value of a quantity related to transport processes (temperature gradient, plasma rotation velocity, etc...), and causes transport through nonlinear processes. The mode disappears as soon as the driving term vanishes.

Regarding the second possibility, the sequence of events could be the following: The starting point is the opening of some poloidally localized radial transport channel (e.g. L-mode filamentary structures [22]). Through this channel, hot and dense plasma is injected into a flux tube on an outer flux surface and moves the plasma out of force balance. This would trigger an MHD wave as a harmonic response of the plasma predominantly with $m=1 / t$. The density on the flux surface equilibrates quickly and the transient MHD mode is damped.

Independent on the direction of causality, our results fit well into the picture: in bad confinement conditions multiple frequency MHD modes are modulated in a correlated way, meaning a radially extended perturbation to the plasma. In good confinement, modes at different frequencies are modulated in an uncorrelated way, therefore the perturbation in the plasma should be mode localized. This difference on the radial extent of the perturbation was also seen in the correlation analysis of electron cyclotron emission (ECE) temperature measurements [4].

The phenomenon seen in shot series \#52147-52152 (Figure 6) can not be easily fit into this picture. It still awaits for a reasonable explanation.

Transient MHD modes and ELM-like modes are not exclusively observed on the W7-AS stellarator. MHD instabilities connected to ELM-like modes have also been reported on the TJ-II stellarator. There, a connection has been found between the presence of the MHD mode in the $10-20 \mathrm{kHz}$ frequency region and a rational surface. Correlation with mm-scale scattering and with radially localized bursts in the plasma potential were also found [23].

\section{Conclusions}

Analysis of W7-AS Mirnov coil signals in a MHD inactive pure ECRH heated plasma was motivated by the observed correlation between the fluctuation of transport relevant quantities and the strength of magnetic fluctuations $[2,4]$. Earlier analyses found that there were bursts in these magnetic signals with a correlation time in the order of $100 \mu s$. These were localized radially [5] and had a poloidal structure [6].

W7-AS Mirnov coil signals were analyzed with the custom developed bandpower correlation method. Steps of the method were: 1. calculation of the continuous spectrogram of the signal, 2. calculation of bandpower signals which approximate the power modulation of the different frequency signal components, 3. correlating different bandpowers. Cross correlation functions (CCFs) show the strength of the statistical 
connection between the modulation of the different frequency modes.

Correlation between the power modulation of fluctuations with different frequencies was found to be strong in 'bad confinement' plasmas and insignificant in the statistical sense in 'good confinement' plasmas. A speculation was presented on the origin of this observation based on the hypothesis that transient MHD modes accompany ELM-like radial transport events [4].

\section{References}

[1] Weller A et al 2001 Phys. Plasmas 8931

[2] Basse NP et al 2005 Phys. Plasmas 12052512

[3] Saffman M et al 2001 Rev. Sci. Instrum. 722579

[4] Zoletnik S et al 2005 Europhysics Conference Abstracts 29C P-5.023

[5] Zoletnik S et al 1999 Phys. Plasmas 64239

[6] Anton M et al 1998 J. Plasma Fusion Research SERIES 1259

[7] Brakel R and W7-AS team 2002 Nucl. Fusion 42903

[8] Mallat S 2001 A Wavelet Tour of Signal Processing (Academic Press)

[9] Zegenhagen S et al 2006 Plasma Phys. Control. Fusion 481333

[10] MPI für Plasmaphysik, Garching 2000 Mirnov Probes MIR-1 at W7-AS (internal report)

[11] Zoletnik S et al 2002 Plasma Phys. Control. Fusion 441581

[12] Takahashi H et al 2002 Nucl. Fusion 42448

[13] Papp G et al 2005 Europhysics Conference Abstracts 29C P-5.021

[14] Mertins A 1999 Signal Analysis (John Wiley \& Sons Ltd)

[15] Gabor D 1946 J. Institute of Electric Engineers 93429

[16] Delprat N et al 1992 IEEE Trans. Inf. Th. 38644

[17] Dudok de Wit T 1996 The URSI Review on Radio Science 1993-1995 CPT-96 3369

[18] Duncan P and Rusbridge MG 1993 Plasma Phys. Control. Fusion 35825

[19] Hirsch M et al 2000 Plasma Phys. Control. Fusion 42 A231

[20] Grigull P et al 1999 Proc. 12th Stellarator Workshop

[21] Hirsch M et al 1998 Europhysics Conference Abstracts 22C 2322

[22] Counsell GF et al 2006 Proc. 21st IAEA Fusion Energy Conference EX/P4-6

[23] Alejaldre C et al 2002 Proc. 19th International Conference on Fusion Energy OV/4-4 